\title{
COEXISTING PROSTATE CANCER FOUND AT THE TIME OF HOLMIUM LASER ENUCLEATION OF THE PROSTATE FOR BENIGN PROSTATIC HYPERPLASIA: PREDICTING ITS PRESENCE AND GRADE IN ANALYZED TISSUE
}

\author{
Naeem Bhojani ${ }^{2}$, Ronald S. Boris ${ }^{1}$, M. Francesca Monn ${ }^{1}$, Jessica A. Mandeville ${ }^{3}$, \\ and James E. Lingeman ${ }^{1}$ \\ ${ }^{1}$ Indiana University School of Medicine, Indianapolis, IN, USA \\ ${ }^{2}$ Centre Hospitalier de l'Université de Montréal, Montréal, Québec, Canada \\ ${ }^{3}$ Lahey Hospital and Medical Center, Burlington, MA, USA
}

\author{
Address all correspondence to: \\ James E. Lingeman M.D. \\ Indiana University School of Medicine \\ 1801 Senate Blvd., Suite 220 \\ Indianapolis, IN 46202 \\ Tel: 317.962 .2485 \\ Fax: 317.962.2893 \\ E-mail: jlingeman@iuhealth.org \\ Word Count: \\ Abstract: 227 \\ Text: 2643 \\ Running head: Prostate cancer after HoLEP \\ Key Words: BPH, Holmium enucleation, prostate, prostate cancer, HoLEP
}

This is the author's manuscript of the article published in final edited form as:

Bhojani, N., Boris, R. S., Monn, M. F., Mandeville, J. A., \& Lingeman, J. E. (2015). Coexisting prostate cancer found at the time of holmium laser enucleation of the prostate for benign prostatic hyperplasia: predicting its presence and grade in analyzed tissue. Journal of Endourology / Endourological Society, 29(1), 41-46.

http://doi.org/10.1089/end.2014.0359 


\begin{abstract}
Objective: To determine the incidence of prostate cancer identified on holmium enucleation of the prostate (HoLEP) specimens and evaluate variables associated with prostate cancer identification.
\end{abstract}

Methods: All patients undergoing HoLEP between 1998 and 2013 were identified. Patients with a known history of prostate cancer were excluded. Multivariable logistic regression assessed variables associated with identification of prostate cancer on HoLEP specimens and Gleason 7 or higher prostate cancer among the malignant cases. Gleason grade was used as a proxy for disease severity. Each of the models was adjusted for age, preoperative PSA, and HoLEP specimen weight.

Results: The cohort was comprised of 1272 patients of whom $103(8.1 \%)$ had prostate cancer identified. Prostate cancer cases had higher pre-HoLEP PSA $(p=0.06)$ but lower HoLEP specimen weight $(p=0.01)$. On multivariate logistic regression, age and preoperative PSA were associated with increased odds of prostate cancer being present $(p<0.01$ each) while increasing HoLEP specimen weight was associated with decreased odds of prostate cancer $(p<0.001)$. Men older than 80 had $20 \%$ predicted probability of being diagnosed with prostate cancer. Seventy-eight percent of prostate cancer cases were Gleason 6 or less. Pre-HoLEP PSA was associated with increased adjusted odds of intermediate or high grade prostate cancer.

Conclusion: Prostate cancer identified by HoLEP is not uncommon but is generally low risk disease. Older patients with smaller prostate glands have the highest odds of prostate cancer identification. 


\section{Introduction}

Benign prostatic hyperplasia $(\mathrm{BPH})$ affects a significant proportion of men in the United States with $70 \%$ of men in their seventh decade demonstrating histologic evidence and $40 \%$ displaying moderate to severe urinary symptoms ${ }^{1}$. Many will undergo active intervention with 912 per 100,000 men in the US undergoing a BPH procedure in $2008^{2}$. While TURP remains the most common active intervention for $\mathrm{BPH}^{2,3}$, a wellestablished alternative is holmium laser enucleation of the prostate (HoLEP). HoLEP, initially described in 1996, reliably provides a more complete resection when compared with TURP, especially for large glands, with a superior safety profile ${ }^{4,5}$. As with TURP, HoLEP is a procedure that provides tissue for pathologic analysis.

Before the adoption of prostate specific antigen (PSA) screening, almost $20 \%$ of prostate cancer was diagnosed from TURP ${ }^{6-8}$. However, since the introduction of PSA screening, this rate has declined significantly to $6-8 \%{ }^{8}$. Previous studies have demonstrated that up to $12 \%$ of men undergoing HoLEP will be found to have prostate cancer in their analyzed tissue ${ }^{9,10}$. Most studies have suggested that prostate cancer identified on HoLEP is generally indolent but some men may ultimately require subsequent treatment of their prostate cancer. In our experience, post-HoLEP robotassisted radical prostatectomy, although feasible with encouraging oncologic outcomes, is a more challenging operation associated with longer functional recovery times compared to historical controls ${ }^{11}$. Thus, identifying men at increased risk for clinically significant prostate cancer is an important consideration in managing patients with BPH disease who are considering enucleation surgery.

Our primary objective was to identify the incidence of prostate cancer identified by HoLEP in men with no history of prostate cancer. Additionally, we sought to determine preoperative variables that can be used to predict the presence of prostate 
cancer in men undergoing HoLEP and to examine the burden of prostate cancer disease in this at risk patient population.

\section{Methods}

A retrospective analysis of patients who underwent HoLEP at IU Health Methodist Hospital between 1998 and 2013 was performed. Patients with a prior history of prostate cancer diagnosis were eliminated from the analysis $(n=34)$. Pre-operative data including age, PSA, transrectal ultrasonography (TRUS) of prostatic volume, and PSA density were collected. Additionally, post-operative data including final specimen weight and pathological tissue findings were recorded. Patients without pre-operative PSA were eliminated $(n=221)$. Patients with elevated PSAs (at the referring urologist's discretion) had negative pre-operative prostate biopsies. Prostate volume as assessed by TRUS was identified for $76 \%$ of patients. PSA density was calculated from preoperative PSA and TRUS prostate volume and was available for $75 \%$ of patients. We acknowledge that the likelihood of identifying prostate cancer may vary between patients with a history of TRUS biopsy and those without; however, to the extent of our documentation, the detection of cancer at the time of HoLEP did not vary between patient populations $(\mathrm{p}=0.314)$.

HoLEP was performed in similar fashion to our previously described technique ${ }^{12}$. Briefly, using a 550 micron end firing laser fiber through a $28 \mathrm{Fr}$ continuous flow resectoscope, the transition zone of the prostate was completely enucleated. Next, using a Lumenis morcellator (Yokneam, Israel), morcellation was completed removing the excised tissue from the bladder and prostatic fossa. The entire HoLEP specimen was submitted for pathologic analysis by dedicated genitourinary pathologists.

The relationship between pre- and post-operative variables and identification of the presence of prostate cancer was assessed using univariate and multivariate logistic 
regression models. A separate multivariate logistic regression model was performed on patients with prostate cancer to evaluate the relationship between preoperative variables and Gleason 7 or higher disease. The variables chosen for univariate analyses were age, pre-operative PSA, pre-operative TRUS volume, final weight of prostate specimen from HoLEP, and percent of prostate resected. For multivariate analyses, only age, preoperative PSA, and the HoLEP specimen weight were included. As TRUS volume was not available for $24 \%$ of patients, we chose to use HoLEP specimen weight in the analysis as it was available for $97 \%$ of patients. Patients with missing data were excluded from the regression analyses. All statistical analyses were performed using Stata version 12.1 (Stata Corp. LP, College Station, TX). In all tests, a two-tailed significance level of $p<0.05$ was used. Institutional Review Board approval was granted for the conduct of this study.

\section{Results}

Patient pre-operative and post-operative characteristics are demonstrated in Table 1. A total of 1272 patients undergoing HoLEP between 1998 and 2013 were analyzed. One hundred and three (8.1\%) patients had prostate cancer identified on final pathological prostatic tissue analysis. Average pre-HoLEP PSA was $7.2 \mathrm{ng} / \mathrm{mL}$ (range: 0.04-121). Of note, two patients had pre-HoLEP PSA values higher than $65 \mathrm{ng} / \mathrm{mL}$ in the benign group $(120,121 \mathrm{ng} / \mathrm{mL})$. Both patients presented with retention and prostatitis prior to HoLEP. Average volume resected during HoLEP was 78.9 grams (SD: 53). The mean percent prostate tissue resected was $84 \%$, which varied between $77 \%$ for the patients with prostate cancer and $85 \%$ among the non-malignant cases. Patients with prostate cancer identified on final pathological analysis were older, had higher preoperative PSA, smaller pre-operative TRUS prostatic volume, and smaller HoLEP specimen weight (Table 1). Length of stay was similar between the two groups. 


\section{Prediction of prostate cancer}

On univariate logistic regression analysis, age and pre-operative PSA were significantly associated with increased odds of identifying prostate cancer on final pathological analysis after HoLEP (Table 2). The weight of the HoLEP specimen was associated with decreased odds of prostate cancer identification in the HoLEP specimen (Table 2). On multivariate logistic regression, age, pre-operative PSA and decreasing specimen weight remained significantly associated with the presence of prostate cancer (Table 2). Figure 1 depicts the relationship between age and identification of prostate cancer when adjusting for pre-operative PSA and HoLEP specimen weight. Patients who were 80 years of age were at $20 \%$ predicted probability of having prostate cancer identified on HoLEP $(<0.001)$.

\section{Prediction of Gleason 7 or higher prostate cancer}

Of the 103 patients with prostate cancer, 80 were Gleason 6 or less (78\%), 14 were Gleason 7 (12 Gleason 3+4; 2 Gleason 4+3), and 9 were Gleason 8 or higher. Univariate regression results are shown in Table 3. On multivariate logistic regression, increasing pre-operative PSA was associated with increased odds of Gleason 7 or greater prostate cancer $(p=0.002)$ (Table 3).

Amongst the 103 patients identified with prostate cancer on HoLEP, follow-up information was available for 48 . Thirty-five of 48 patients (73\%) were followed with active surveillance and had received no treatment for their diagnosed prostate cancer at last follow-up. Twelve of the 48 patients (25\%) underwent some form of primary therapy: 7 received external beam radiation, 3 underwent radical prostatectomy, and 2 received hormonal therapy. One patient with advanced disease underwent bilateral orchiectomy. A total of three patients from this cohort had developed metastatic prostate cancer. At last follow-up, three patients from the surveillance cohort had died of non- 
prostate cancer related causes while two men with metastatic disease in the intervention cohort had died.

\section{Discussion}

Prior reports have identified the majority of prostate cancer in analyzed HoLEP tissue to be primarily low grade, indolent disease that has not required subsequent intervention. A recent study by Rivera et al suggested that more than $90 \%$ of patients found to have prostate cancer at the time of HoLEP were managed successfully with active surveillance ${ }^{13}$. Previous authors have reported a strong correlation between preoperative PSA and TRUS volume ${ }^{9,14}$. Similar to simple prostatectomy for BPH, PSA values drop dramatically following HoLEP ${ }^{14,15}$ and subsequent increases in PSA-velocity are associated with increased risk of prostate malignancy ${ }^{9}$. The PSA kinetics of patients discovered to have prostate cancer after HoLEP have not been well studied, although it would be reasonable to hypothesize that increases in PSA in this patient population may represent disease progression as the primary PSA-producing transition zone has been completely eliminated. In our study, pre-operative PSA was the only variable associated with intermediate and high grade prostate cancer and it was significantly higher in the prostate cancer than benign cohorts. As demonstrated in Figure 1, men older than 70 years with smaller prostates were at the greatest risk of harboring prostate cancer and may warrant further workup prior to HoLEP.

A recent study by Zlotta et al found that $40 \%$ of men over 60 and nearly $60 \%$ of men over 80 had prostate cancer on autopsy specimen ${ }^{16}$. Over $40 \%$ of the prostate cancer identified in this older group was found in the transition zone which is the primary prostatic tissue removed during HoLEP ${ }^{16}$. These results contrast with our finding of only $8 \%$ incidental prostate cancer in our HoLEP patients. Despite this discordance, low rates of prostate cancer in HoLEP specimens was recently reported by Rivera et al who 
cited a $13 \%$ cancer rate amongst their HoLEP patients ${ }^{13}$. Differences in comparison with historical autopsy series may be at least partially attributable to the vast majority of HoLEP patients having regular PSA checks or, in some cases, pre-operative prostate biopsies. Prior publications have suggested an association between $\mathrm{BPH}$ and prostate cancer that may be inflammatory mediated, even suggesting that the presence of BPH may increase the risk of malignancy ${ }^{17}$. In contrast, our results suggest a potentially protective effect of $\mathrm{BPH}$ and obstructive symptoms on the presence of prostate cancer. Whether this represents a true cause and effect phenomenon or merely isolating a prescreened patient population remains unknown. In any event, future research exploring the relationship between $\mathrm{BPH}$ and prostate cancer should be considered.

It is well established that the risk of prostate cancer increases with age. Because $\mathrm{BPH}$ is the primary contributor to PSA rise and, as previously discussed, BPH may impact the incidence of prostate cancer, the relationship of prostate cancer in aging men with $\mathrm{BPH}$ is less understood. Despite the fact that we report a significant increase in the adjusted odds of prostate cancer detection for each decade of life over 60 (Figure 1), we did not find that there was an association between age and Gleason score. Although the relationship between age and prostate cancer was anticipated based on prior research, it does provide valuable support that the relationship between prostate cancer and age remains present even in men with co-existing $\mathrm{BPH}$ and obstructive symptoms, although it does not appear to impact the clinical significance of malignancy.

Unlike the positive association between age and the presence of prostate cancer, the relationship between prostate size and prostate cancer has previously been shown to be inverse ${ }^{18}$. Similar to prior findings, our cohort again demonstrated a decrease in the odds of prostate cancer identification as prostate gland size increased beyond $30 \mathrm{~g}$. Prior studies have hypothesized that the seemingly protective effect of larger glands harboring prostate cancer may be hormone mediated or related to a longer time before a 
nodule penetrates the prostate capsule ${ }^{19}$. Additionally, pre-operative PSA, TRUS volume, and PSA density were all significant on univariate analysis. These findings suggest that prostate volume and PSA density may be important predictors of the presence of prostate cancer in men with $\mathrm{BPH}$ undergoing HoLEP and that these tests should be considered prior to surgery, particularly in men with higher risk of prostate cancer detection.

Although the limitations of PSA are well documented, PSA continues to be widely used as both a screening tool and a method of detecting prostate cancer recurrence ${ }^{20,21}$. Our results suggest that there is value in obtaining a PSA pre-operatively for men with BPH who are scheduled to undergo HoLEP. All patients with an elevated PSA should be considered for prostate biopsy or repeat prostate biopsy before surgery. If localized prostate cancer is identified prior to HoLEP, some of these men may elect to pursue alternative procedures to address both $\mathrm{BPH}$ and prostate cancer concomitantly.

Predicting the biologic behavior of prostate cancer found at HoLEP remains challenging. As documented in the past on autopsy and whole mount tissue studies, typically only $20-40 \%$ of prostate cancer originates in the prostate transition zone ${ }^{16,22}$. Therefore, our data may be under-representing the true incidence and volume of disease present and is in no way a perfect surrogate for predicting prostate cancerrelated risk. Despite this, interpretation of our results does allow us to make relevant observations regarding prostate malignancy in patients with $\mathrm{BPH}$ undergoing complete transition zone enucleation: 1) HoLEP candidates who are older men with small glands are more likely to harbor cancer in their enucleated tissue. More specifically, independent of PSA and specimen weight, an estimated $20 \%$ of men who are at least 80 years old will have prostate cancer identified on HoLEP specimen; 2) the number of patients with prostate cancer in this cohort is low (less than $10 \%$ ) with predominantly low grade disease; and 3) pre-operative PSA may be predictive of both presence and 
aggressiveness of disease. Significantly, we have limited follow-up for our patients who were identified with prostate cancer on HoLEP as only 48 of the 103 continued to receive care at our institution. As we are a tertiary referral center, this is not unexpected; however, it does limit conclusions that can be drawn regarding the prognosis of patients diagnosed with prostate cancer from HoLEP specimen.

The majority of patients were outside referrals and cancer management was often directed by the referring provider and not captured in our database. For our internal HoLEP patient population diagnosed with prostate cancer, a general discussion of options was standard. Patients with low risk disease were recommended surveillance with a baseline PSA at six weeks followed by serial PSA checks and digital rectum exams every six months. Patients with a rising PSA or an abnormal exam were offered restaging TRUS biopsy and then treatment if indicated. Patients with intermediate and/or high risk disease post-HoLEP were staged appropriately and then offered definitive management, surgery vs radiation (external beam or IMRT) vs radiation and androgen deprivation typically without restaging biopsy.

Ultimately, the creation of a treatment and surveillance algorithm for managing this patient population based on numerous pre-operative variables is warranted. Similar treatment flowcharts for prostate cancer and non-HoLEP related BPH procedures are well documented ${ }^{23}$. Future studies that evaluate PSA kinetics in patients with prostate cancer identified by HoLEP will aid in determining appropriate follow-up recommendations for this unique population. In our population of post-HoLEP patients with negative final prostate pathology, we anticipate PSA levels to fall below 1 . If levels do not nadir below 1 or rise quickly in follow-up, we recommend repeat TRUS prostate biopsy as suspicion for prostate cancer is high. In the present study, our data offers potentially useful counseling data for patients prior to HoLEP regarding the risk of 
prostate cancer being identified, as well as predicting higher risk disease in this patient population.

Our study has inherent limitations. It is retrospective and the number of prostate cancer patients is relatively low. Additionally, because a high percentage of our patients are referred from outside facilities, pre-operative TRUS techniques likely demonstrated inter-operator variability and TRUS volume was missing for a significant proportion of patients. Because $24 \%$ of TRUS volumes were missing, we used HoLEP specimen weight in all of our analyses. Additionally, TRUS biopsy was not standardized amongst referring physicians and this variable was not completely captured in our database. As a tertiary referral center, some post-HoLEP patients are lost to follow-up and therefore information on prostate cancer treatment initiation was not available for the entire cohort. It is possible that a disproportionate number of patients who required subsequent therapy received follow-up at our institution, thus creating a selection bias in the descriptive findings that we present. Despite the stated limitations, our study represents one of the largest analyzed cohorts of prostate cancer in HoLEP patients.

\section{Conclusion}

The existence of incidentally detected prostate cancer at the time of HoLEP in men with $\mathrm{BPH}$ and obstruction is low and the majority of patients will have low grade disease. Men over 70 with smaller glands are at the highest risk of harboring prostate cancer in their analyzed tissue. Optimal treatment and surveillance strategies for management of men with prostate cancer after HoLEP need to be established. 


\section{References}

1. McVary KT: BPH: epidemiology and comorbidities. Am J Manag Care 2006; 12: S122-128.

2. Malaeb BS, Yu X, McBean AM, et al.: National trends in surgical therapy for benign prostatic hyperplasia in the United States (2000-2008). Urology 2012; 79: 1111-1116.

3. Lowrance WT, Southwick A, Maschino AC, et al.: Contemporary practice patterns of endoscopic surgical management for benign prostatic hyperplasia among urologists in the United States. J Urol 2013; 189: 1811-1816.

4. Gilling PJ, Cass CB, Cresswell MD, et al.: Holmium laser resection of the prostate: preliminary results of a new method for the treatment of benign prostatic hyperplasia. Urology 1996; 47: 48-51.

5. Gilling PJ, Kennett K, Das AK, et al.: Holmium laser enucleation of the prostate (HoLEP) combined with transurethral tissue morcellation: an update on the early clinical experience. J Endourol 1998; 12: 457-459.

6. Helfand BT, Mongiu AK, Kan D, et al.: Outcomes of radical prostatectomy for patients with clinical stage T1a and T1b disease. BJU Int 2009; 104: 304-309.

7. Meeks JJ, Maschino AC, McVary KT, et al.: Clinically significant prostate cancer is rarely missed by ablative procedures of the prostate in men with prostate specific antigen less than $4 \mathrm{ng} / \mathrm{ml}$. J Urol 2013; 189: 111-115.

8. Mai KT, Isotalo PA, Green J, et al.: Incidental prostatic adenocarcinomas and putative premalignant lesions in TURP specimens collected before and after the introduction of prostrate-specific antigen screening. Arch Pathol Lab Med 2000; 124: 1454-1456.

9. Elmansy HM, Elzayat EA, Sampalis JS, et al.: Prostatic-specific antigen velocity after holmium laser enucleation of the prostate: possible predictor for the assessment of treatment effect durability for benign prostatic hyperplasia and detection of malignancy. Urology 2009; 74: 1105-1110.

10. Nunez R, Hurd KJ, Noble BN, et al.: Incidental prostate cancer revisited: early outcomes after holmium laser enucleation of the prostate. Int J Urol 2011; 18 : 543-547.

11. Suardi N, Scattoni V, Briganti A, et al.: Nerve-sparing radical retropubic prostatectomy in patients previously submitted to holmium laser enucleation of the prostate for bladder outlet obstruction due to benign prostatic enlargement. Eur Urol 2008; 53: 1180-1185.

12. Kuo RL, Paterson RF, Kim SC, et al.: Holmium Laser Enucleation of the Prostate (HoLEP): A Technical Update. World J Surg Oncol 2003; 1: 6.

13. Rivera ME, Frank I, Viers BR, et al.: Holmium Laser Enucleation of the Prostate and Perioperative Diagnosis of Prostate Cancer: An Outcomes Analysis. J Endourol 2014 Epub.

14. Tinmouth WW, Habib E, Kim SC, et al.: Change in serum prostate specific antigen concentration after holmium laser enucleation of the prostate: a marker for completeness of adenoma resection? J Endourol 2005; 19: 550554. 
15. Humphreys MR, Miller NL, Handa SE, et al.: Holmium laser enucleation of the prostate--outcomes independent of prostate size? J Urol 2008; 180: 24312435.

16. Zlotta AR, Egawa S, Pushkar D, et al.: Prevalence of prostate cancer on autopsy: cross-sectional study on unscreened Caucasian and Asian men. J Natl Cancer Inst 2013; 105: 1050-1058.

17. De Nunzio C, Kramer G, Marberger M, et al.: The controversial relationship between benign prostatic hyperplasia and prostate cancer: the role of inflammation. Eur Urol 2011; 60: 106-117.

18. Al-Azab R, Toi A, Lockwood G, et al.: Prostate volume is strongest predictor of cancer diagnosis at transrectal ultrasound-guided prostate biopsy with prostate-specific antigen values between 2.0 and $9.0 \mathrm{ng} / \mathrm{mL}$. Urology 2007; 69: 103-107.

19. Gershman B, Dahl DM, Olumi AF, et al.: Smaller prostate gland size and older age predict Gleason score upgrading. Urol Oncol 2011; 31: 1033-1037.

20. Stamey TA: The central role of prostate specific antigen in diagnosis and progression of prostate cancer. J Urol 1995; 154: 1418-1419.

21. Stamey TA: Preoperative serum prostate-specific antigen (PSA) below 10 microg/l predicts neither the presence of prostate cancer nor the rate of postoperative PSA failure. Clin Chemistry 2001; 47: 631-634.

22. McNeal JE, Redwine EA, Freiha FS, et al.: Zonal distribution of prostatic adenocarcinoma. Correlation with histologic pattern and direction of spread. Am J Surg Pathol 1988; 12: 897-906.

23. Capitanio U, Briganti A, Suardi N, et al.: When should we expect no residual tumor (pT0) once we submit incidental T1a-b prostate cancers to radical prostatectomy? Int J Urol 2011; 18: 148-153.

List of Abbreviations

HoLEP Holmium laser enucleation of the prostate

$\mathrm{BPH} \quad$ Benign prostatic hyperplasia

PSA Prostate specific antigen

TRUS Transrectal ultrasonography 
Table 1: Holmium laser enucleation of the prostate: patient characteristics

\begin{tabular}{|c|c|c|c|}
\hline & Benign & Malignant & p-value \\
\hline $\mathrm{N}$ & 1169 & 103 & \\
\hline Age (years), mean (SD) & $70.0(8)$ & $74.5(9)$ & $<0.001$ \\
\hline Pre-operative PSA (ng/ml), mean (range) & $7.0\left(0.04-121^{\mathrm{a}}\right)$ & $9.8(0.13-61)$ & 0.057 \\
\hline $\begin{array}{l}\text { Pre-operative TRUS volume }{ }^{b}(g) \text {, mean } \\
\text { (range) }\end{array}$ & $101.4(9-391)$ & $85.2(23-231)$ & 0.012 \\
\hline PSA density ${ }^{\mathrm{b}}$, mean (range) & $0.08(0.0-1.3)$ & $0.19(0.0-6.6)$ & $<0.001$ \\
\hline \multicolumn{4}{|l|}{ Final specimen weight, $\mathrm{n}(\%)$} \\
\hline$<30 \mathrm{~g}$ & $191(17)$ & $29(28)$ & \multirow{4}{*}{0.005} \\
\hline $30-100 \mathrm{~g}$ & $602(53)$ & $57(55)$ & \\
\hline $100-150 \mathrm{~g}$ & $233(21)$ & $12(12)$ & \\
\hline$>150 \mathrm{~g}$ & $105(9)$ & $5(5)$ & \\
\hline Percent prostate resected, mean (SD) & $84.9(41)$ & $77.0(29)$ & 0.115 \\
\hline Length of stay (hours), mean (range) & $29.3(1-600)$ & $33.8(1-264)$ & 0.233 \\
\hline $\begin{array}{l}6 \text { month post-HoLEP PSA }(\mathrm{ng} / \mathrm{mL}) \text {, mean } \\
\text { (range) }\end{array}$ & $1.2(0.04-66)$ & $4.5(0.1-64)$ & $<0.001$ \\
\hline
\end{tabular}

Abbreviations: SD, standard deviation; TRUS, transrectal ultrasound p-value represents Pearson's chi-squared test or the Mann-Whitney test

${ }^{a}$ Two patients in the benign group had pre-HoLEP PSAs higher than $65 \mathrm{ng} / \mathrm{mL}$. Both of these patients were admitted with retention and prostatitis.

${ }^{b}$ TRUS volume not available for $24 \%$ of cases, PSA density not available for $25 \%$ of cases 


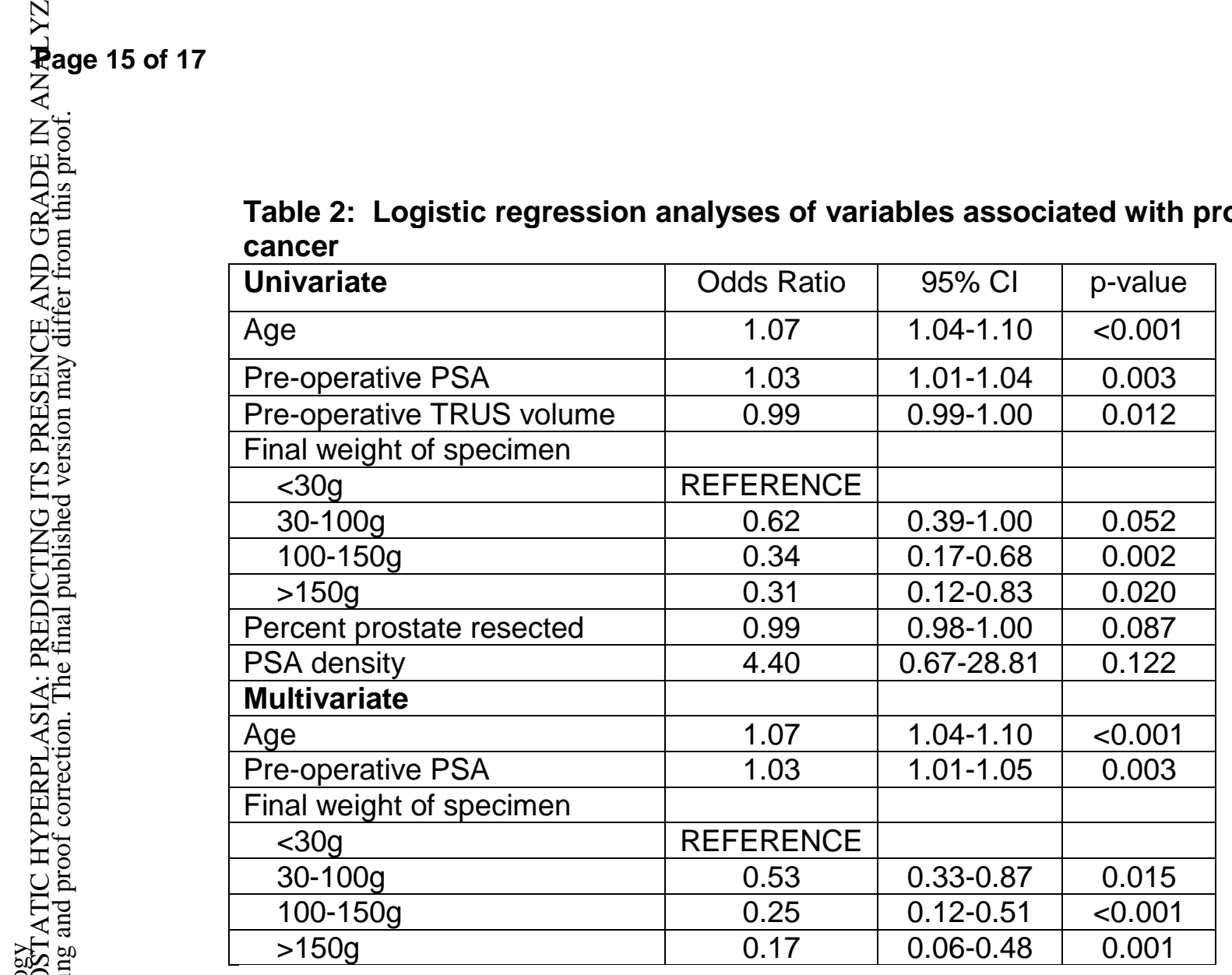
cancer 
Table 3: Variables associated with Gleason Score of 7 or higher in patients with prostate cancer

\begin{tabular}{|l|c|c|c|}
\hline Univariate & Odds Ratio & $95 \% \mathrm{Cl}$ & p-value \\
\hline Age & 1.03 & $0.97-1.09$ & 0.318 \\
\hline Pre-operative PSA & 1.08 & $1.03-1.14$ & 0.001 \\
\hline Preoperative TRUS volume & 1.00 & $0.98-1.01$ & 0.810 \\
\hline Final weight of specimen & & & \\
\hline$<30 \mathrm{~g}$ & REFERENCE & & \\
\hline $30-100 \mathrm{~g}$ & 1.25 & $0.42-3.67$ & 0.688 \\
\hline $100-150 \mathrm{~g}$ & 0.77 & $0.13-4.47$ & 0.768 \\
\hline$>150 \mathrm{~g}$ & 0.96 & $0.09-10.23$ & 0.972 \\
\hline Multivariate & & & \\
\hline Age & 1.01 & $0.95-1.08$ & 0.661 \\
\hline Pre-operative PSA & 1.08 & $1.03-1.14$ & 0.002 \\
\hline Final weight of specimen & & & \\
\hline$<30 \mathrm{~g}$ & REFERENCE & & \\
\hline $30-100 \mathrm{~g}$ & 1.24 & $0.33-4.68$ & 0.750 \\
\hline $100-150 \mathrm{~g}$ & 0.78 & $0.11-5.51$ & 0.805 \\
\hline$>150 \mathrm{~g}$ & 0.41 & $0.03-6.10$ & 0.521 \\
\hline
\end{tabular}




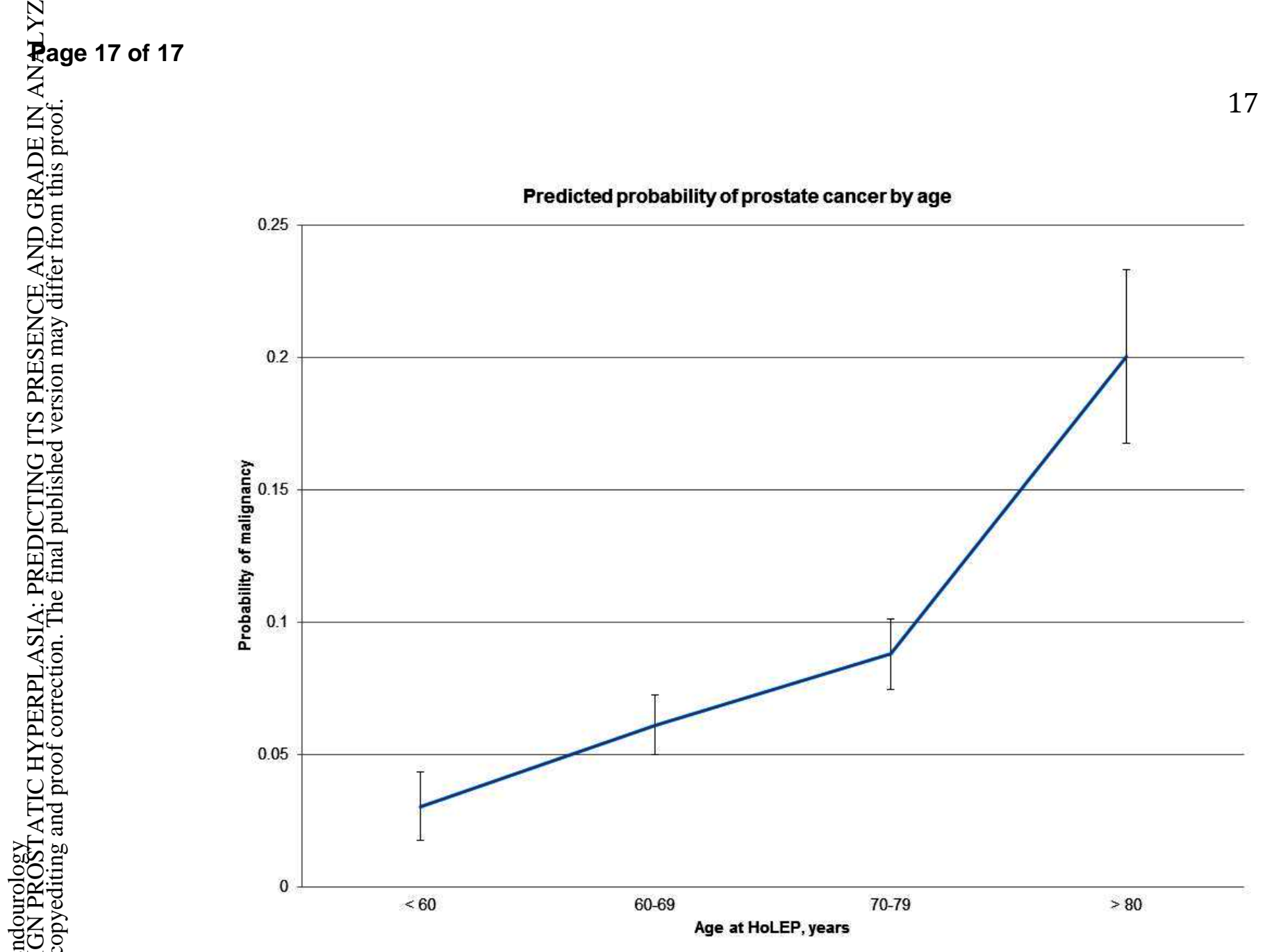

닝 